\title{
Bluff body flow-induced noise control with sliding plasma actuators
}

\author{
VINOGRADOV Igor $^{1} \&$ HUANG Xun ${ }^{1,2 *}$ \\ ${ }^{1}$ Department of Mechanics and Aerospace Engineering, Peking University, Beijing 100871, China; \\ ${ }^{2}$ Department of Aeronautics and Astronautics, Peking University, Beijing 100871, China
}

Received May 5, 2011; accepted June 17, 2011

\begin{abstract}
Sliding plasma actuators were used to control the flow-induced broadband noise radiating from a bluff body. The structure consisted of a cylinder and a torque link that was installed on the lee side of the cylinder. The objective was to reduce the noise generated during the cylinder wake impinging on the torque link. By manipulating cylinder wake with the externally-imposed body force generated by the plasma actuators, the flow-structure interaction was reduced, leading to an attenuation of the broadband noise up to $3 \mathrm{~dB}$ in overall sound pressure level at a free stream speed of $30 \mathrm{~m} / \mathrm{s}$.
\end{abstract}

aeroacoustics, bluff body, plasma actuators

Citation: Vinogradov I, Huang X. Bluff body flow-induced noise control with sliding plasma actuators. Chinese Sci Bull, 2011, 56: 3079-3081, doi: 10.1007/ s11434-011-4687-5

Plasma actuators have recently been extensively studied for their potential in flow active control [1-3]. Simplicity and absence of any moving mechanical parts make plasma actuators a promising option for flow and flow-induced noise control applications [4]. The history and principle of plasma actuators can be found in [5] and references therein. In this work, a sliding plasma actuator, a type exploiting dielectric barrier discharge (DBD) [6], and its flow control applications are of particular interest. Through ionization, the plasma actuators generate weakly-ionized atmospheric plasma consisting of charged particles moving within a coupled electric field. Owing to collisions between charged and ambient particles, plasma actuators act as a jet along the actuator surface [7]. The maximally-induced jet velocity is up to $8 \mathrm{~m} / \mathrm{s}$ measured at $20 \mathrm{~mm}$ away from the electrodes [8].

Recent work [8,9] has demonstrated the potential of DBDs in the attenuation of tonal noise from a cavity. The focus of this work is specifically on the investigation of broadband noise attenuation with plasma actuators. Previous work had involved a single cylinder at Reynolds numbers between $1 \times 10^{4}$ and $4 \times 10^{4}[10,11]$. It was found that the

*Corresponding author (email: huangxun@pku.edu.cn) cylinder wake width can be controlled and vortex shedding from the cylinder suppressed. In the present work, the attenuation of broadband noise generated by a more complex bluff body geometry (Figure 1), representing practical structures such as a simplified landing gear component, is investigated using near-field hot-wire probes and far-field microphones.

The geometry tested consists of a cylinder and a torque link component. The torque link, installed downstream from the cylinder, is formed from two triangle-shaped plates at a variable angle. The cylinder is made of a foam material to prevent electric interference with the plasma actuators. The torque link component is constructed from aluminum.

The noise radiating from the structure is higher at broadband frequencies compared with a single cylinder [10,11]. This increase is thought to be caused by the flow-structure interaction between the cylinder wake and the torque link. Figure 2 shows the layout of a so-called sliding plasma actuator installed on the surface of the cylinder (see Figure 3) [5], where the thick black curves denote the electrodes made by copper tapes of thickness $0.04 \mathrm{~mm}$. The sliding plasma actuator was selected here for its better flow control performance compared with a typical DBD plasma actuator. In particular, Figure 2 shows a relatively uniform and stable 

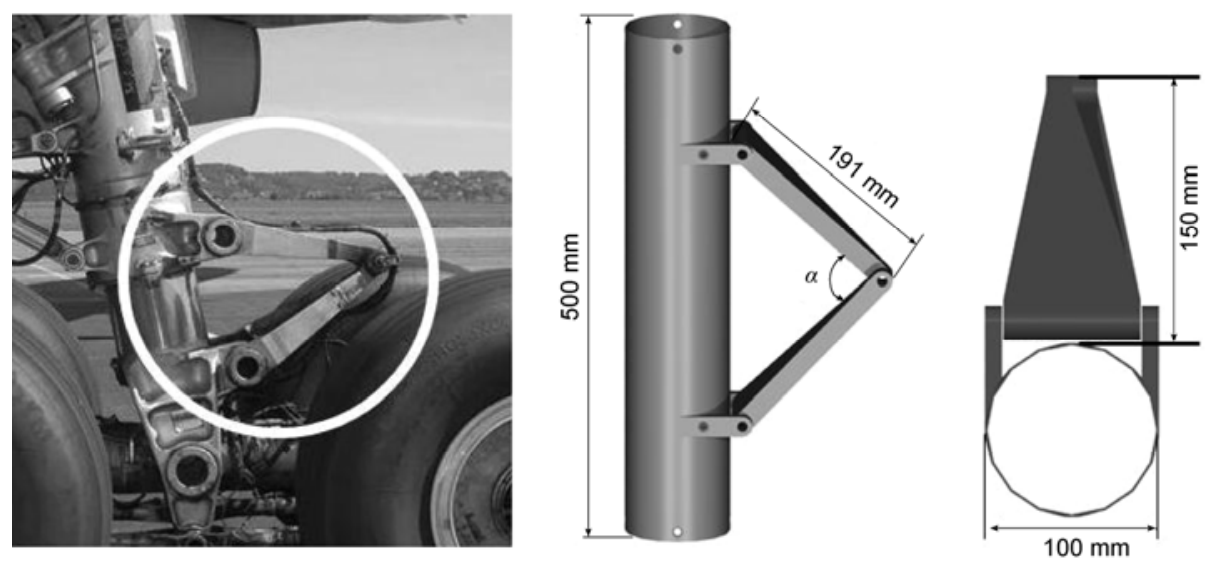

Figure 1 Schematic of the testing model and a practical application.
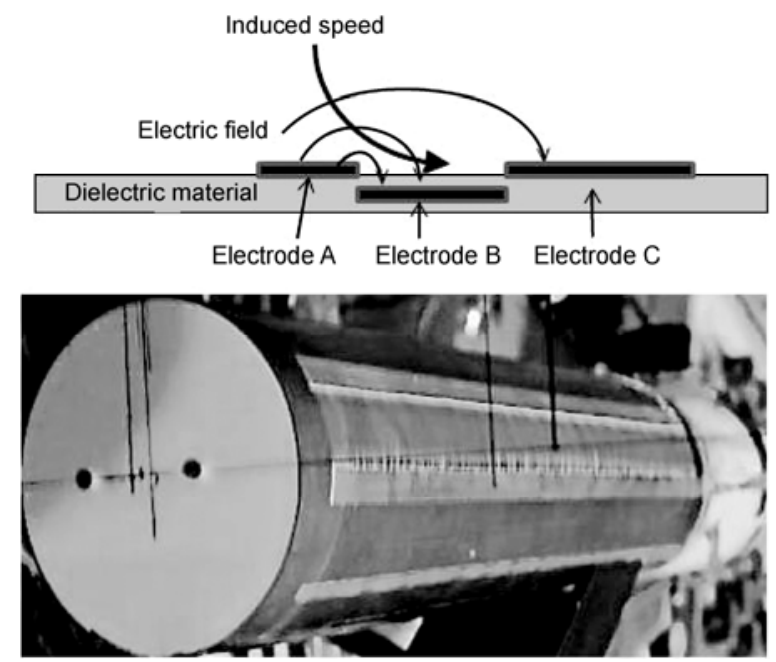

Figure 2 Schematic of a sliding plasma actuator system.

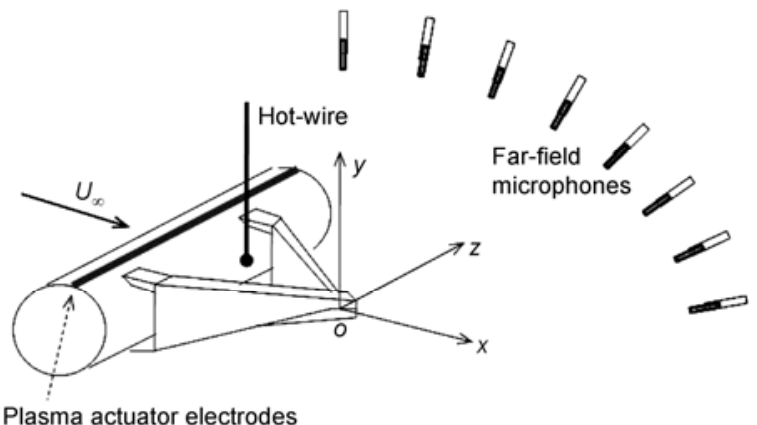

Figure 3 Setup of the experiment units.

plasma sheet covering a large amount of the cylinder surface.

The width of each electrode exposed to air is $10 \mathrm{~mm}$ whereas that insulated by the dielectric material is $20 \mathrm{~mm}$. Both parameters are selected empirically; silicon rubber of thickness $1 \mathrm{~mm}$ constitutes the dielectric material. The length of each plasma actuator is $350 \mathrm{~mm}$, which is consistent with the size of the test section. Electrode A is con-

nected to a high voltage alternating current (AC) source, electrode B is grounded, and electrode C is connected to a high voltage direct current (DC) source. The peak-to-peak $\mathrm{AC}$ voltage is $25 \mathrm{kV}$ and the $\mathrm{DC}$ voltage is $10 \mathrm{kV}$. The overall power consumption is about $200 \mathrm{~W}$. As shown in Figure 3, the exposed electrodes are placed at almost $\pm 90^{\circ}$ with respect to the oncoming flow, and the velocity induced from the plasma is directed upstream, and thus behaves like a virtual fairing that can presumably reduce the flow-structure interaction and consequently attenuate flow-induced noise.

The flow control mechanisms were examined in an anechoic chamber facility. A nozzle $(500 \mathrm{~mm} \times 350 \mathrm{~mm})$ connecting to a plenum chamber can produce a jet flow at a speed $\left(U_{\infty}\right)$ of $30 \mathrm{~m} / \mathrm{s}$. The corresponding Reynolds number based on the cylinder diameter $D$ is $2.1 \times 10^{5}$. It is still impossible to visualize the flow field downstream of the cylinder after the installation of the torque links using the present two-dimensional Particle Image Velocimetry (PIV) system. Hot-wire probes were used at $(x, y, z)=(-100,20,0)$ $\mathrm{mm}$ and $(x, y, z)=(-100,40,0) \mathrm{mm}$. Figure 4 shows the velocity fluctuations in the cylinder wake, where the solid lines denote results under plasma actuation, whereas the dash lines relate to the result with an inactive plasma. It can be seen that the flow fluctuations are reduced by almost 10 $\mathrm{dB}$ under plasma actuation, which confirms the aforementioned flow control mechanisms. A similar finding was found in previous experiments [10] although only for an isolated cylinder at lower Reynolds numbers. It is also worthwhile mentioning here that the three-dimensional PIV measurements and/or simulations with a suitable numerical model for plasma actuation effects [12-14] have to be considered to reveal details of the wake structures.

Figure 5 shows the overall sound pressure level (OASPL) results that were measured using far-field microphones placed almost $20 D$ away from the model, where $D$ denotes the cylinder diameter. The distance is mainly limited by the size of the test facility. The OASPL results were computed between $100 \mathrm{~Hz}$ and $10 \mathrm{kHz}$; beyond that range the self- 

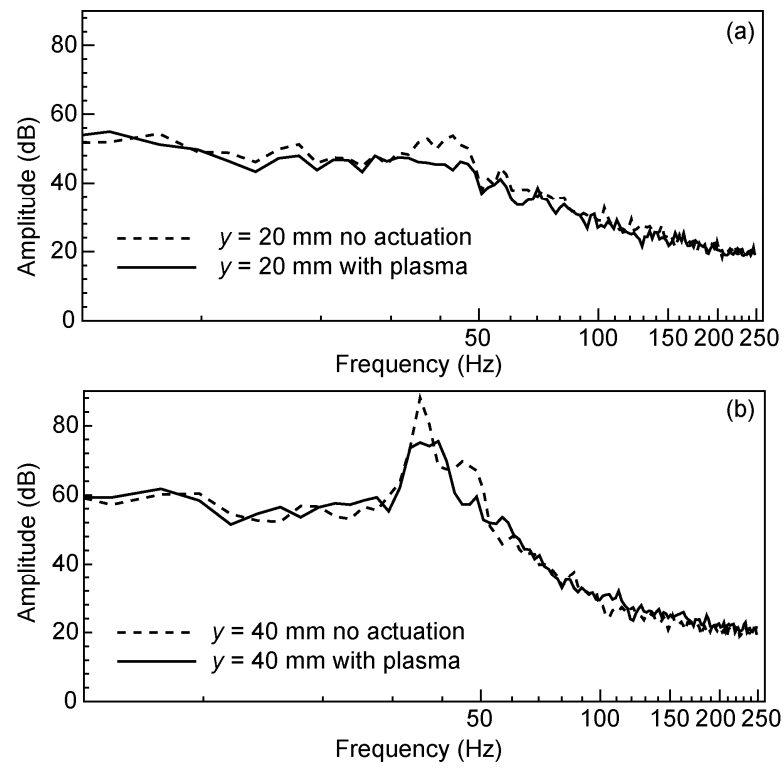

Figure 4 Hot-wire results achieved at $(x, y, z)=(-100,20,0) \mathrm{mm}$ (a) and $(x, y, z)=(-100,40,0) \mathrm{mm}(\mathrm{b})$.

noise from the plasma is strong and has to be omitted here. Figure 5 shows that at $U_{\infty}=30 \mathrm{~m} / \mathrm{s}$ the upstream-directed plasma actuation can reduce OASPL noise by almost $3 \mathrm{~dB}$, which is presumably caused by the aforementioned flow

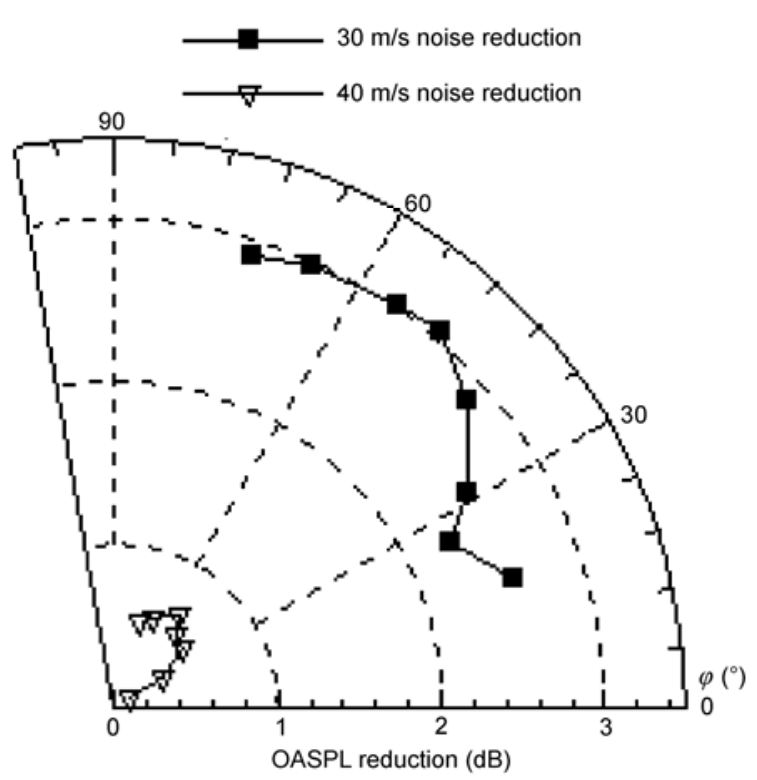

Figure 5 Noise reductions measured with far-field microphones. control mechanisms. More specifically, as the flow-structure interaction at the trailing edge components in cylinder wake can be reduced with upstream-directed plasma actuation, the flow-induced noise radiated from the trailing edge components can consequently be attenuated. At low angles, it seems that the control effects are reduced, which is understandable as the related microphones are located in the fluctuated model wake. In contrast, the control effects deteriorate at higher free stream speeds. Noise reduction has been reduced by less than $1 \mathrm{~dB}$ at $U_{\infty}=40 \mathrm{~m} / \mathrm{s}$. This relatively low control effectiveness at high speed reflects the main shortcoming of DBD plasma actuators used nowadays, and a search for improvements remains an open problem for the area.

In summary, this experimental investigation studied the control effectiveness of the plasma actuators on broadband noise radiated from a bluff body. It showed that the flow fluctuation between the cylinder and the trailing components can be effectively manipulated with sliding plasma at $U_{\infty}=30 \mathrm{~m} / \mathrm{s}$. As a result, flow-induced noise radiating from the structure can be reduced. However, the effectiveness of the plasma actuators deteriorated at higher speeds. To further reduce broadband noise, additional reduction of the interaction between the cylinder wake and the trailing components could be achieved by improving the plasma actuator performance, an aspect which is ongoing.

The experimental part of this work was conducted at the University of Southampton in the UK; the second author would like to thank Prof. Xin Zhang for discussion and guidance. This work was supported in part by the China Postdoctoral Science Foundation (20100470151) and the National Natural Science Foundation of China (90916003, 11150110134 and 11050110109).

1 Do H, Kim W, Cappelli M A, et al. Appl Phys Lett, 2008, 92: 071504

2 Li G, Xu Y J, Lin B, et al. Sci China Ser E-Tech Sci, 2009, 52: 37153721

3 Zhang P F, Liu A B, Wang J J. Sci China Tech Sci, 2010, 53: 27722782

4 Huang X, Zhang X. Int J Aeroacoust, 2010, 9: 679-704

5 Moreau E. J Phys D: Appl Phys, 2007, 40: 605-636

6 Roth J R. Phys Plasmas, 2003, 10: 1166-1172

7 Kim W, Do H, Mungal M G, et al. Appl Phys Lett, 2007, 91: 181501

8 Huang X, Zhang X. Phys Fluids, 2008, 20: 037101

Huang X, Chan S, Zhang X, et al. AIAA J, 2008, 46: 241-250

10 Sung Y, Kim W, Mungal M G, et al. Exp Fluids, 2006, 41: 479-486

1 Forte M, Jolibois J, Pons J, et al. Exp Fluids, 2007, 43: 917-928

12 Peers E, Huang X, Luo X F. IEEE Trans Plasma Sci, 2009, 37: 2250-2256

13 Li X, Weng C S. Chinese Sci Bull, 2009, 54: 1641-1647

14 Peers E, Ma Z K, Huang X. Phys Lett A, 2010, 374: 1501-1504

Open Access This article is distributed under the terms of the Creative Commons Attribution License which permits any use, distribution, and reproduction in any medium, provided the original author(s) and source are credited. 